\title{
GUTOPHAGY - ADAPTIVE MOLECULAR MECHANISMS IN CONDITION OF STARVATION
}

\author{
Małgorzata Tomasiak, Beata Cichacz, Agnieszka Pedrycz \\ Departament of Histology and Embryology Medical University in Lublin, Poland
}

\section{ABSTRACT}

Autophagy is an extremely old process during which long-lived proteins and cellular organelles are removed by means of lysosomes. Autophagy may be caused by cellular stress mechanisms. Research has proven that autophagy plays a key role in obtaining nutrients and adapting to the conditions of starvation. Owing to this, it takes part in maintaining homeostasis in cytoplasm and cell nucleus. This objective may be achieved through a number of ways Depending on the manner in which a substrate connects with the lysosome, we can talk about macroautophagy and microautophagy. Additionally, some authors also distinguish a chaperone-mediated autophagy. The article presented below describes molecular mechanisms of each type of autophagy and focuses particularly on macroautophagy, which is the best understood of all the autophagy types. Key words: autophagy, cellular starvation, lysosome, cell death.

ARTICLE INFO

PolHypRes 2015 Vol. 52 Issue 3 pp. 71-75

ISSN: $1734-7009$ elSSN: 2084-0535

DOI: $10.1515 / \mathrm{phr}-2015-0018$

Pages: 5, figures: 0 , tables: 0

page www of the periodical: www.phr.net.pl

Publisher

Polish Hyperbaric Medicine and Technology Society
Rewiev article

Delivery date: $05.06 .2015 \mathrm{r}$.

Date of approval for print: 20.07 .2015 r. 


\section{INTRODUCTION}

Autophagy is one of the morphological types of cell death. The term autophagy was introduced in the 1960s by Christian de Duve. It is derived from the Greek word means 'self-eating' [1]. In terms of phylogenetics it is a very old process which probably first appeared a billion years ago in one-cell organisms in the time of their adaptation to periods of limited food availability [2]. Autophagy may be caused by stress-inducingmechanisms for the cell, such as starvation, pathogenic infection, hypoxia or the presence of oxygen radicals. One of the characteristic features of the discussed process is the participation of lysosomes in protein degradation. It enables the removal of long-lived protein from the cell. Autophagy contributes to the degradation of superfluous or damaged organelles. The products retrieved in the procedure are reused as building and energetic material. Owing to these properties autophagy enables adaptation of the organism in the event of starvation. Other functions that have so far have been linked with this process include: it takes part in the synthesis of the surfactant on the surface of type II pneumocytes, maturation of erythrocytes and biosynthesis of neuromelanin in dopaminergic neurons.

Literature provides several types of autophagy. Depending on the manner in which a substrate connects with the lysosome, we can talk about macroautophagy and microautophagy. Additionally, some authors also distinguish a chaperone-mediated autophagy.

Macroautophagy was the first to be characterised in mammals as the main source of aminoacids and other basic components that are required by the organism during times of starvation. In the course of macroautophagy entire regions of the cytoplasm, which contain different organelles, are surrounded with a double or multiple membrane resulting in the formation of enclosed vacuoles called autophagosomes [3]. The phagosomal membrane may originate in the Golgi apparatus, endoplasmic reticulum, cell membrane or mitochondrion. Another step is the fusion of these vacuoles together with the lysosomes which results in the release of hydrolytic enzymes, while the ph of the interior drops, which is conducive to the material contained inside. The structure thus formed is called the autophagolysosom.

The responsibility for the genetic regulation of autophagy in humans is held by the AUT and ATG genes. 27-30 Atg1-Atg30 proteins take part in the process of autophagy $[4,5]$. These proteins can be divided into four groups.

- Atg/UKL1/2 serine/threonine protein kinase regulated with mTOR kinase (mammalian Target of Rapamycin). Hormonal signals and the deficit of aminoacids induce autophagy which is controlled by the mTOR-dependent signal path). The inhibition is brought about during the combination of the GTP particle with the Gi3 protein trimer, whereas combination with GDP stimulates sequestration of cytoplasmic components [6].

- The complex of PI3K/Vps34 lipid kinases mediates in the nucleation of vesicles i.e. creation of the autophagosome vesicle membrane. It also takes part in the intracellular movement of the cytoskeleton elements and the substrate sequestration to autophagic vacuoles.

- The Atg12-Atg5 ubiquitin-like conjugation system.

- $\quad$ The Atg8-PE ubiquitin-like conjugation system. Ubiquitin-like complexes mediate in the growth of vesicles. Two transmembrane proteins, mAtg9 and VMP1, take part in the recirculation of Atg proteins [7].

The serine/threonine kinase (mTOR), using aminoacids and growth factors, affects cell growth regulation, transcription, translation, formation of rRNA and ribosomes as well as a proliferation and cell movement.

The lack of nutrients leads to the inhibition of TOR activity inducing various cellular responses such as the inhibition of cellular growth in the G1 phase, inhibition of protein synthesis, changes in protein transcription and the activation of the UKL1 protein kinase.

Autophagy is regulated by the contrasting mTOR and UKL1 kinase activity. Inhibiting mTOR with rifamycin increases the activity of UKL1 kinase, whereas the mTOR activation through the RHEB protein strongly inhibits UKL1 activation [8].

ULK1 and PI3K affect the initiation of the process of autophagy as a component of the larger protein complex [8]. These kinases affect the activation of additional ATG proteins that are located on phagophore membranes and thus contribute to their maturation.

In the course of induction, a complex of Atg13 and $\operatorname{Atg} 101$ is formed in the phagophore membrane by the Atg1, which is responsible for its elongation. It is this protein complex that is negatively regulated by the TOR [9]. Atg1 is also responsible for the Atg9 phosphorylation. The activated Atg9 protein is indispensable for an effective recruitment of Atg8 and Atg18 when forming the autophagosome [10].

The responsibility for the initiation of the process lies also with Beclin 1 which is encoded by the BECN1 gene. This is a protein which, together with phosphatidylinositol triphosphate kinase (PI3K), is involved in the transportation of substrates to autophagic vacuoles. This complex can be found in one part of the Golgi apparatus which indicates that the function of the complex is to control autophagy by supplying phosphatidylinositol triphosphate from the Golgi apparatus to isolating membranes (Lamparska-Przybysz 2005). The Beclin 1 activity must not be inhibited by the presence of Bcl-2 and Bcl-X anti-apoptotic proteins.

During the formation of the autophagosome, two conjugated systems are created. One of these engages four ATG 5,7,10,12 proteins, and the other is a combination of a phosphatidylethanolamine with the Atg8-Aut7 complex [11].

The human Atg8 homologue is the LC-3 protein (MAP1LC3 - microtubule-associated proteins 1A/1B light chain 3). We distinguish two forms of this protein: The LC3-I found in cytoplasm, whose number is variable in different cell types, and LC3-II are connected with the autophagosome surface and correlate with their number [12]. The Atg4 proteasis, during the post-translational modification leads to the dissociation of 22 amino acid fragments at the C-end on the light chain of the LC-3 protein, and thus exposing the remainder of the 
glycine. The Atg7 and Atg3 catalyse reactions with the participation of the Atg12-Atg5-Atg16L complex that lead to the LC3-I conjugation with the phosphatidylethanolamine (PE) via the exposed glycine remainder. The LC3II thus formed is capable of connecting with the external and internal membrane of the autophagosome. The process of converting LC3I into the LC3II form is enhanced after the induction of autophagy.

The LC3II that is already present on the phagophore membrane connects with the p62/SQTM1 protein which takes part in the loading into the autophagosome and is combined with the components intended to be degraded [13]. The substrate together with the LC3 is moved inside the autophagosome. Products of digestion that takes place in the autolysosome are released back to cytoplasm. After the process of autophagy, the level of LC3II and p62/SQTM1 inside the lysosome decreases. The LC3II in the cytosol is released by the Atg4B and reconverts into LC3 I. LC-3II is a reliable molecular marker of autophagy [6]. The fluorescence labelled LC3 may be observed in the form of small points which are the forming autophagosomes.

The absorption by way of cytosol endocytosis via lysosomes is called microautophagy. The degradation of the macromolecules contained in it is effected through small bulgings of the lysosomal membrane. The formation of autophagosomes is not required. This type of autophagy plays a more important role in selective degradation of organelles. Pexophagy takes place in peroxisomes and may only be effected through micro or macroautophagy.

Chaperone-dependent autophagy requires the presence of appropriate receptors on the lysosome membrane. In order for the protein intended for degradation to be bound with the receptor, the participation of cytosolic chaperones is required; it is the chaperone-substrate complex that binds with the receptor present on the lysosome membrane. Another chaperone is responsible for transmitting the substrate inside the lysosome. In the amino acid sequence of each protein that is a substrate, there is a KFERQ fragment (Lys-Phe-Glu-Arg-Gln) that directs it to the lysosome.

The motif is recognised by the cytosolic chaperone, which is a heat shock protein hsc73. The hsc73 substrate complex that is formed next connects with the Lamp2q receptor placed on the lysosome membrane. The chaperone, which is now located inside the lysosome, also contributes to the translocation of the protein from the surface of the membrane inside where the substrate is subjected to hydrolysis.

Autophagy is a non-selective process which evenly encompasses all cytoplasmic macroparticles. Mitochondria, peroxisomes and the fragments of the Golgi apparatus are also decomposed. Autophagy enables the maintenance of hemostasis in the cytoplasm and the cell nucleus. It is also regarded to be a cellular strategy for survival during times when the access to nutrients has been restricted.

Most of the components that are decomposed in the autophagolysosome become a source of nutrients that enable cellular survival with the least possible energy consumption.

Liver cells are classic examples as autophagy begins in times of hunger in order to produce amino acids that are converted to glucose and supplied to the organs that demand it the most, such as the brain, and to erythrocytes [14]. Autophagy disorders lead to pathologies such as neurodegeneration caused by the accumulation of protein aggregation, muscle damage caused as a result of the accumulation of autophagosomes that weakens cell function; neoplasms [15].

\section{BIBLIOGRAPHY}

1. Allyson L., Anding, Eric H. Baehrecke.: Autophagy in Cell Life and Cell Death. www.sciencedirect.com/science/article/pii/S0070215315000484, 2015

2. Baran P.: Death types analysis of liver hepatocytes in pregnant female rats induced by the preeclampsia imposed on the nephrotic syndrome. Doctoral thesis 2013

3. Clausen T.H., Lamark T., Isakson P., Finley K., Larsen K.B., Brech A., Øvervatn A., Stenmark H., Bjørkøy G., Simonsen A., Johansen T.: p62/SQSTM1 and ALFY interact to facilitate the formation of p62 bodies/ALIS and their degradation by autophagy. Autophagy, 6: 330-344; 2010:

4. Dunlop E.A, Tee A.R. :.mTOR and autophagy: A dynamic relationship governed by nutrients and energy. Semin Cell Dev Biol, 36: 121-129; 2014:

5. Kamada Y., Funakoshi T., Shintani T., NeganoK., Oshumi M., Oshumi Y.: Tor-mediated induction of autophagy via an Apg1 protein kinase complex. J Cell Biol. , 150: 1507-1513; 2000:

Ketteler R., Seed B.: Quantitation of autophagy by luciferase release assay. Autophagy, 4: 801-806; 2008 :

7. Klionsky D.J.: Autophagy revisited: a conversation with Christian de Duve. Autophagy. 4: 740-743; 2008:

8. Klionsky D., Gregg J., Dunn W., Emr S., Sakai Y., Sandoval I., Sibirny A., Subramani S., Thumm M., Veenhuis M.: A unified nomenclature for yeast autophagy-related genes. Dev Cell. 5: 539-545,2003

9. Lamparska-Przybysz M., Motyl T.: Autophagy: a tool for survival or death of a tumour cell. Post Biol Kom. 32: 13-22; 2005:

10. Luneman JD., Munz C.: Autophagy in CD4+ T-cell immunity and tolerance. Cell death different.16: 79-85; 2009:

11. Ohsumi Y.: Mollecular dissection of autophagy:two ubiquitin-like systems. Nat.Rev.Mol. Cell. Biol., 2:211-216, 2001

12. Ostrowski K; Histologia ed. II 667-669,1995

13. Papinski D., Schusching M., Reiter W., Wilhelm L., Barnes C. A., Maiolica A., et.all: Early steps in autophagy depend on direct phosphorylation of Atg9 by the Atg1 kinase. Mol Cell, 53: 471-483; 2014:

14. Polewska J.: Autophagy- molecular mechanizm, apoptosis and cancer. Post Hig Med Dosw; 66: 921-936; 2012:

15. Reggiori F., KilionskyD.J.: Autophagy in the Eukaryotic Cell. Eucaryotic Cell. 1: 11-21; 2002:

16. Rudnicka K.W., Szczęsna E., Miszczyk E., Mikołajczyk-Chmiela M.: Apoptosis and Autophagy - mechanisms and methods of detection. Post Biol Komórki 38: $247-265 ; 2006:$

17. Świderek E, Strządała L.: Autophagy and the BNIP3 protein in neoplasms. Advances in Hygiene and Experimental Medicine, 67: $363-370 ; 2013$

prof. dr hab. n. med. Agnieszka Pedrycz

Departament of Histology and Embryology Medical University in Lublin

ul. Radziwiłłowska 11 20-080, Lublin e-mail: apw4@wp.pl 